Décadrages Décadrages

cinéma, à travers champs Cinéma, à travers champs

15 | 2009

Raoul Ruiz

\title{
La dixième édition du Festival international du film fantastique de Neuchâtel
}

Freddy Landry

\section{OpenEdition}

1 Journals

Édition électronique

URL : https://journals.openedition.org/decadrages/126

DOI : $10.4000 /$ decadrages. 126

ISSN : 2297-5977

Éditeur

Association Décadrages

Édition imprimée

Date de publication : 1 octobre 2009

Pagination : 88-99

ISBN : 978-2-9700668-0-4

ISSN : 2235-7823

Référence électronique

Freddy Landry, " La dixième édition du Festival international du film fantastique de Neuchâtel », Décadrages [En ligne], 15 | 2009, mis en ligne le 26 novembre 2012, consulté le 03 avril 2022. URL http://journals.openedition.org/decadrages/126 ; DOI : https://doi.org/10.4000/decadrages.126 


\section{La dixième édition du Festival international \\ du film fantastique de Neuchâtel}

\section{Par Freddy Landry}

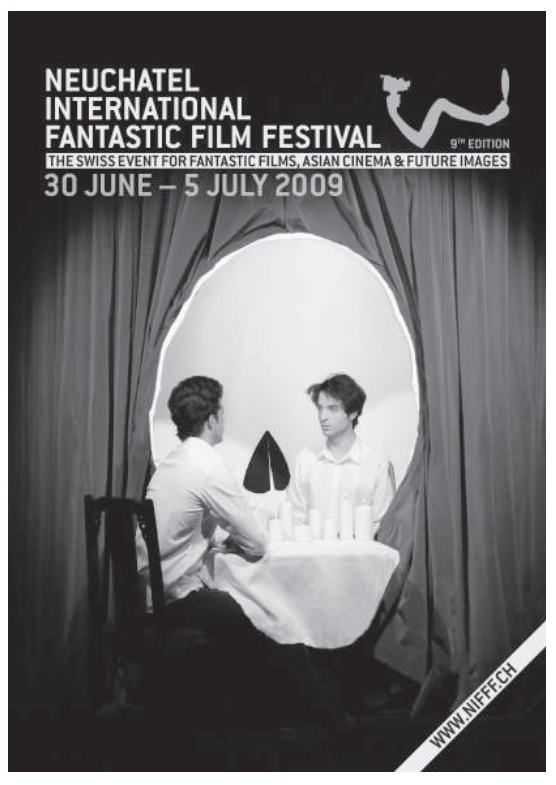

\section{NIFFF : présentation}

Né en Suisse francophone dans une ville qui compte un peu plus de trente mille habitants - le double avec sa banlieue -, le «Neuchâtel International Fantastic Film Festival» ( NIFFF) fêtera sa dixième édition en 2010 (après des débuts en 2000 avec relâche en 2001). Projet utopique il y a dix ans, le festival est devenu aujourd'hui une manifestation reconnue.

Lors de la première édition, 5000 places furent vendues au cours d'une manifestation ayant coûté un peu plus de deux cent mille francs. En 2009, dans le triplex de l'Apollo, au théâtre du Passage et en Plein Air, 22000 places le furent grâce à un budget d'un million et 400000 francs environ. Locarno 2009 annonce 160000 entrées pour un budget d'un peu plus de 11 millions. Le rapport budget/siège du NIFFF est ainsi de presque cinquante francs en 2000 et d'environ soixante en 2009, alors que Locarno 2009 s'approche de septante. Le NIFFF semble s'être d'emblée inscrit dans des normes helvétiques.

Dès la première édition, la volonté de présenter le fantastique sous plusieurs angles se manifeste, allant du gore pur et sans humour, avec son rouge qui n'a rien à voir avec le sang et ses excès de violence gratuite, jusqu'à la suggestion poétique des surréalistes en passant par l'humour, l'étrangeté, la provocation et la discrétion de récits conventionnels. On évoque le "cinéma de genre", mais il est difficile d'éviter la notion d'auteur, même si le réalisateur est parfois remplacé ou associé au scénariste, au producteur ou au responsable des effets spéciaux.

L'édition 2009, du 30 juin au 5 juillet, offre une centaine de projections, rencontres diverses, séminaires (comprenant des Ouverture et Clôture avec discours), Open Air, compétitions de longs et de courtsmétrages européens avec désignation de candidats uniques au Méliès européen, compétition asiatique et de courts métrages suisses, rétrospective William Castle - illusionniste visionnaire, une "Catégorie III, cinéma de l'excès et de la subversion ", des sueurs froides en découvrant le 
cinéma de genre scandinave contemporain , une approche de «L'étrange selon Shinji Aoyama» (voir dans ce numéro, l'article d'Alain Boillat), un "spécial Screenings", un "made in NE» et enfin un symposium "Imagine the Future».

Le cinéphile le plus acharné arrivera à suivre cinq ou six projections ou manifestations par jour, le tiers de l'offre. Le curieux qui se satisfait de trois visites quotidiennes, assistera à une quinzaine de séances. Choix est ici fait de s'arrêter sur les sueurs froides provoquées par le cinéma scandinave. Sur le site de la revue (www.decadrages.ch), nous prendrons également en considération l'Open Air destiné au grand public, la censure qui sévit à Hong-Kong (voir la «catégorie III»), la place du courtmétrage et la vitalité des séries télévisées.

Pour aborder la première décennie d'un festival destiné à perdurer, nous avons conduit des discussions avec Olivier Müller, fondateur et ancien président du NIFFF, Anaïs Emery, cofondatrice et directrice artistique, Pierre-Yves Jeanneret, cofondateur et actuel président, ainsi que Mathieu Béguelin, trait d'union entre films et invités, films et spectateurs. Parallèlement aux collaborateurs rémunérés, quelques dizaines de bénévoles assurent aussi le bon fonctionnement du festival.

\section{Scandinavie}

Peut-on "mesurer» les lignes de force d'un festival à travers les prix et mentions décernés depuis le début par les différents jurys confondus? En neuf éditions, les jurys NIFFF ont par exemple retenu treize longs métrages d'Asie, dont neuf films japonais, signalant sans surprise le rôle de pionnier de cette cinématographie. Onze titres européens ont été retenus, dont six films scandinaves. Le NIFFF avec d'autres a attiré l'attention sur un phénomène intéressant.

\section{Survol historique}

Le fantastique scandinave a un passé que les cinéphiles des débuts de la seconde moitié du siècle dernier associent à de fort lointains souvenirs (de vision): Mauritz Stiller et Le Trésor d'Arne (Suède, 1919), Victor Sjöström pour La Charrette fantôme (Suède, 1922), qui doit à Bergman et ses Fraises sauvages (Suède, 1957) de n'être pas oublié comme réalisateur, ou encore Benjamin Christensen (La Sorcellerie à travers les âges, Danemark, 1918) ou Carl Theodor Dreyer (Vampyr, France/Allemagne, 1932), pour ne citer que quelques exemples. Toute l'œuvre de Bergman, dès la fin des années 1950, est parsemée de fantastique souvent poétique et surréaliste. Lars von Trier a aussi contribué à enrichir le genre, depuis les années 1980, par exemple avec The Kingdom (1994), une étrange série 
télévisée "pointue». Le fantastique scandinave contemporain est donc ancré dans un passé qui connut de réels bonheurs, surtout dans sa dimension métaphysique, mystique ou poétique. Le récent Millénium de Nils Oplev (Suède/Danemark, 2009) qui a fait correcte carrière en 2009 dans les salles européennes, jouera-t-il pour d'autres films de Scandinavie le rôle de locomotive, en particulier avec son délirant personnage féminin?

Jean-Luc Godard, dans un "Bergmanorama» paru dans Les Cahiers du cinéma en janvier 1958 ( $\mathrm{n}^{\circ}$ 143), ne fut pas le seul à souligner l'importance de ce cinéaste un peu trop oublié aujourd'hui. Et pourtant, au début des années 1960, nous n'étions guère qu'une petite dizaine dans une salle de Locarno pour y découvrir un matin La Nuit des forains (Suède, 1953) lors d'une rétrospective!

\section{Quatre films scandinaves}

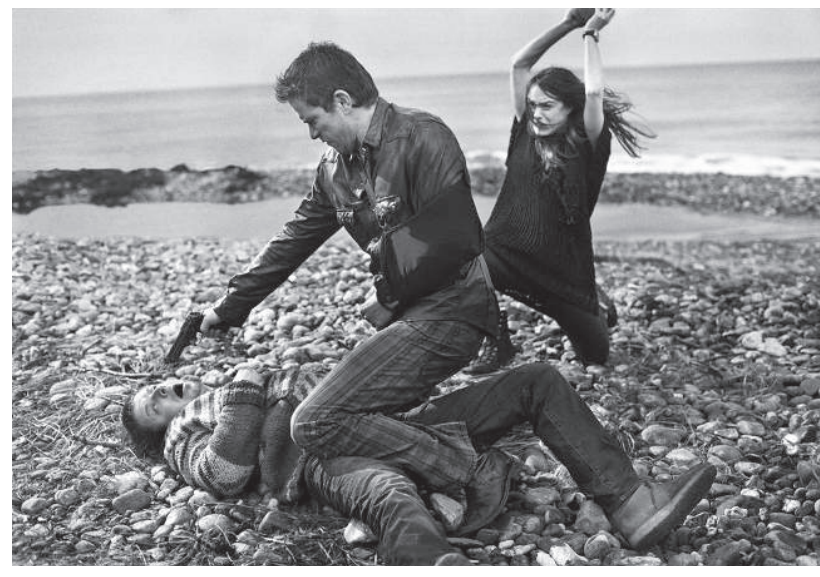

Just Another Love Story (Ole Bornedal, Danemark, 2009) Jonas, un brave père de famille se trouve être plus ou moins responsable d'un accident qui envoie une jeune femme, Julia, dans le coma. Il lui rend visite à

\section{Sauna (Antti-Jussi Annila, Finlande, 2008)}

A la fois scénaristes, réalisateurs, acteurs, monteurs, producteurs, les frères Mika, l'aîné, et Aki Kaurismaki sont à peu près les seuls contemporains à être connus hors de la Scandinavie. Ils ne sont pas les seuls l'hôpital de temps en temps. Sortie du coma, d'abord amnésique, Julia n'empêche pas sa famille de prendre Jonas pour son petit ami. Celui-ci décide, par curiosité, par ennui, par goût de la découverte, d'entrer dans ce jeu, qui l'entraîne de plus en plus loin.

Mais il faut bien finir un film: ce qui était récit réaliste d'un fantasme, d'une erreur de jugement, d'une usurpation d'identité dont il faudrait saisir les mobiles des uns et des autres, vire de bord en abandonnant la démarche psychologique.

On est dans un film de genre, d'abord un peu esquivé. Par convention, il faut y revenir. Un massacre va se produire au bord de l'eau, comme s'il était indispensable de verser dans l'horreur sanguinolente. Ce virage a quelque chose d'artificiel. Dans un film d'horreur, il faut de l'horreur même gratuite. A moins que tout ce qui précède cette violence ne soit indispensable pour l'amplifier.

à faire du cinéma en Finlande. Encore faut-il faire des rencontres dans un festival ou l'autre, pas forcément aisées avec des versions originales sous-titrées seulement en anglais.

Dans Sauna, les extérieurs créent un sentiment 
d'hostilité dans une grande forêt aux arbres inquiétants. Aux abords d'une zone marécageuse et boueuse, un village surgit du néant. Deux personnages d'un petit groupe, l'un géographe, l'autre soldat s'en veulent d'avoir laissé mourir un homme et sa fille dans des conditions exécrables. On sent d'emblée peser sur le film un sentiment de culpabilité renforcé par les paysages. En même temps, il faut s'interroger sur l'Histoire: cela se passe en 1595, à la fin d'une guerre entre la Finlande et la Russie, alors qu'une commission doit délimiter la frontière entre les deux pays. Où placer ce village qui n'existe sur aucune carte? Près du village existe un bloc de béton presque géométrique peint en blanc et qui sera le lieu d'horreur finale, un sauna complètement irréaliste, comme l'était le monolithe de Kubrick dans 2001: L’ Odyssée de l'espace (Etats-Unis, 1968).

Plongés en état de presque totale innocence dans ce film, le besoin se fait ressentir chez le spéctateur d'en savoir davantage sur cet OVNI fort intéressant. Le réalisateur en est à son deuxième film. Il a détourné la commande initiale qui aurait dû montrer un tueur en série agissant dans l'espace d'un sauna. Il en a fait un film historique, sans que l'on sache s'il a pris des libertés avec l'Histoire ou s'il en profite pour évoquer une situation plausible à la fin d'une guerre. Le film a connu une belle carrière dans des manifestations spécialisées depuis le début de 2008, où furent élaborés des dossiers bien documentés sur ce film de genre. La force des images a donc un sens, le sentiment de culpabilité qui plane sur le film va conduire à des attitudes conduisant à des affrontements où vont s'installer foi ou soif de rédemption. Mais en pleine forêt, ce bloc blanc d'un sauna introduit un malaise total par une sorte de négation du temps historique.

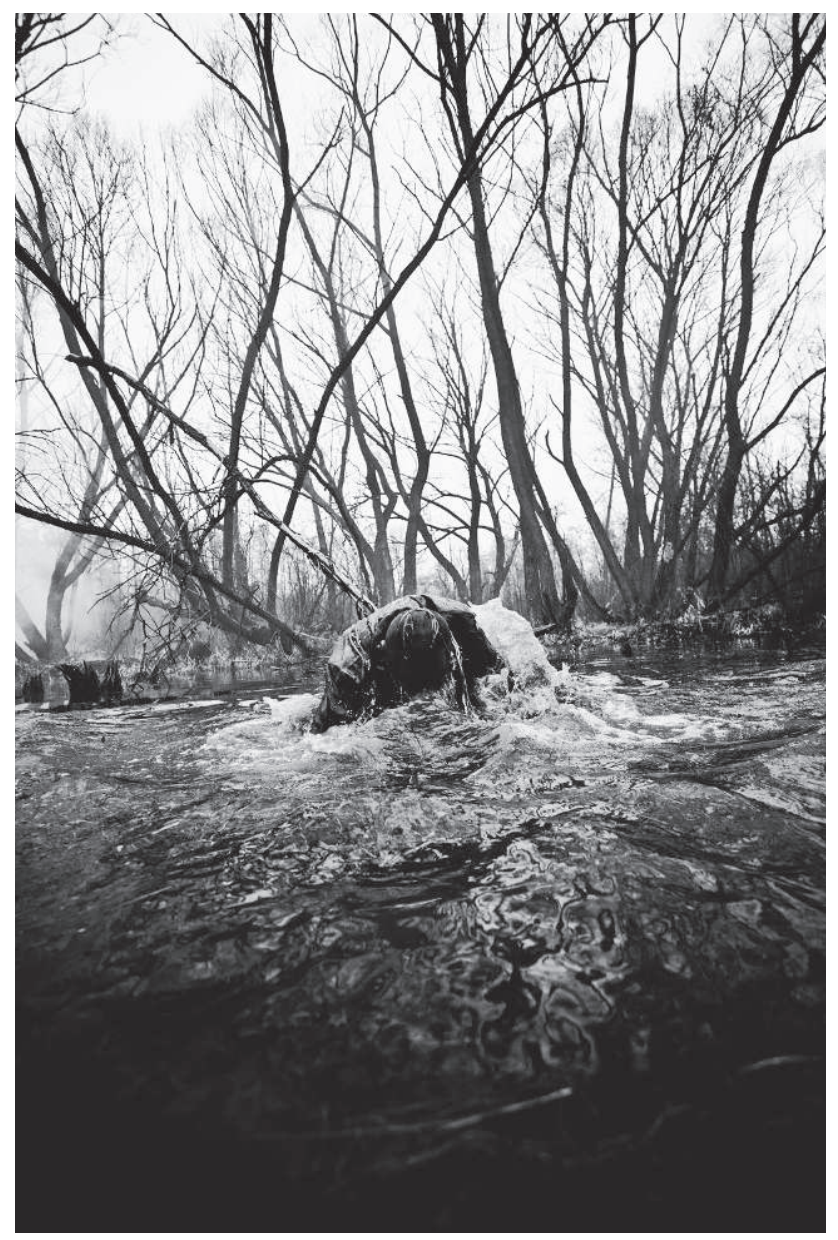

Ici aussi, les brutalités finales ne sont pas d'un grand intérêt, comme dans le film précédent. Mais les portes ouvertes par Sauna, plausibles, sont plus nombreuses et plus intéressantes. A moins d'être dupe d'un film de genre inattendu...

Fear Me Not (Kristian Levring, Danemark, 2009)

Mikael, 40 ans, apparemment bien dans sa peau, accepte de jouer les cobayes pour une firme qui expérimente un anti-dépresseur. Au bout de quelques temps, l'expérience cesse: il y a trop d'effets secon- daires inattendus! Mikael continue d'avaler son stock de pilules. Peu à peu, l'ambiance familiale se trouble: prend-il un malin plaisir à torturer sa femme Sigrid et à étonner sa fille Selma, aux comportements parfaitement normaux? Il exprime son attirance que 


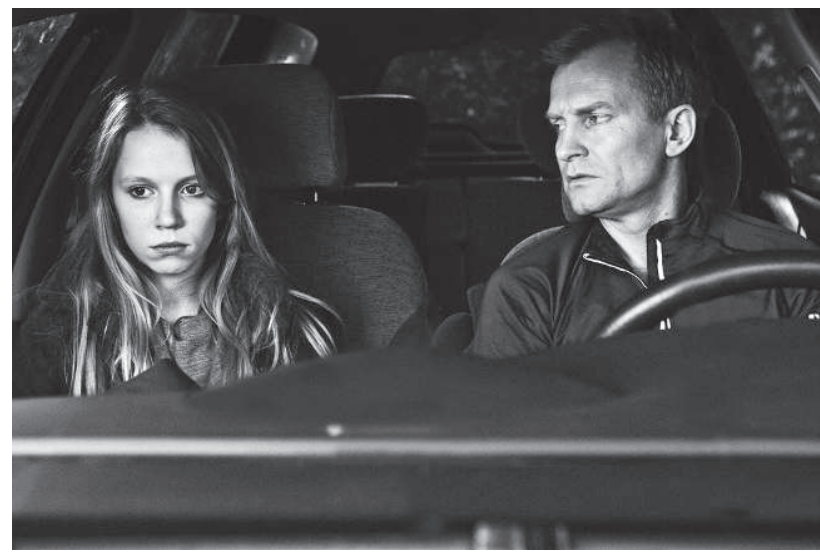

l'on devine ancienne pour la femme de son meilleur ami. Après un bref séjour dans une maison familiale déserte, il prend en stop une jeune fille dont il exige qu'elle lui montre ses seins. Le comportement de Mikael est de plus en plus étrange: sombre-t-il dans la folie? Introduit-il des mensonges dans ses réponses pour troubler ses proches? Cherche-t-il à les priver de leur sérénité ? Est-il diabolique au point de s'apprêter à commettre un meurtre? Il ne changera même pas de comportement quand il apprend que, lors de l'expérience scientifique, il appartenait au groupe auquel on donnait un placebo. Il n'y a ni goutte de sang, ni monstre, ni fantômes, ou d'effets spéciaux, simplement, un esprit quidérape.

Donc pas de scène d'horreur visible, loin de là. Fear Me Not crée avec une force intense une angoisse profondément troublante avec rien ou presque. A plusieurs reprises et chaque fois sous un angle diffé-

\section{Antichrist - Lars von Trier - Danemark}

Pas très bien accueilli à Cannes en mai 2009, Antichrist a choqué. A en croire la presse, le film semblait n'être qu'une suite de violences physiques et morales exercées sur le mari et d'automutilations insupportables de l'épouse sur elle-même. On sentait bien qu'une large partie de la critique, embarrassée, s'en tirait par une double dérobade, soit crier au scandale, soit rent, Mikael avale ses pilules aussi abruptement que le Dr House, souvent face à lui-même dans un miroir. La rapidité des gestes témoigne d'une volonté de ne pas se faire surprendre. Chaque prise conduit pourtant à une détérioration de son comportement.

On peut faire un film intense presque sans action. Les intérieurs sont souvent monochrome; Mikael, bien en évidence dans le cadre, relègue souvent les autres personnages en bordure de l'image. Les extérieurs sont d'une grande beauté aux couleurs sereines, comme si la nature était un élément apaisant aussitôt contredite par le retour en intérieurs ou le métal d'une carrosserie de voiture. Les couleurs, la teinte dominante, les limites du cadre participent ainsi de la mise en scène.

On pense souvent à Bergman. Dans une certaine mesure, l'acteur principal qui apparaît presque dans tous les plans, Ulrich Thomsen, a cette force qui fut celle de l'acteur Max von Sydow qui, même sans rien faire, donnait l'impression d'agir. Dans Festen (Danemark, 1998) de Vinterberg, il était le fils de famille qui brisait le tabou du silence, dans Adams Aebler (Danemark, 2005) le néo-nazi en phase de reconstruction chez un pasteur socialement engagé. Beau pouvoir d'adaptation à des personnages différents.

Le coscénariste du réalisateur, Anders Thomas Jensen, est aussi le réalisateur et scénariste d'Adams Aebler. Un groupe de cinéastes, issus de "Dogma 95", s'attacherait-il à réhabiliter la mise en scène de films fantastiques scandinaves? Le NIFFF, sans dérouler le tapis rouge, propose aux spectateurs un coup de projecteur sur une école vivante.

poser et se poser de multiples questions sur Charlotte Gainsbourg et son interprétation.

Dans un rôle rendu difficile par ses excès et ses exhibitions, elle est tout simplement remarquable. Le prix d'interprétation obtenu à Cannes est mérité. Son partenaire Willem Dafoe aurait tout aussi bien pu être récompensé. On peut mal supporter une excision, une meule sur son axe plantée dans une jambe: cela 
ne suffit pas pour accuser Lars von Trier de médiocrité ou de misogynie.

Antichrist, avec deux «i» et un étrange «t» final comme il est dessiné sur l'affiche, est un film important. Alors que l'épouse (CG) et son mari psychiatre (WD) font l'amour sous la douche, leur enfant quitte sa chambre, ouvre une fenêtre et tombe dans le vide. Elle se sent responsable de cette mort. Son mari tente, en professionnel, mais maladroitement, de l'aider à s'en sortir. Il parvient à lui faire dire en quel lieu elle se sent le plus mal: à l'Eden, leur chalet dans une dense forêt! Ils s'y rendront comme en pèlerinage. A la douleur vont s'ajouter le deuil puis le désespoir, titres respectifs de trois chapitres placés entre prologue et épilogue partiellement ressemblants.

Une clef esthétique aide peut-être à «lire» ce film : le travail sur la couleur, avec le bleu qui va rester constamment inquiétant, bleu de la nuit, bleu d'un bouquet de fleurs, bleu des habits portés par l'un et l'autre. Le vert de la nature d'une dense forêt vouée au sabbat de sorcières mentales l'accompagnera, analysé du reste lors d'un dialogue. Un manteau jaune, un feu orangé, une lumière blanche, le rouge du sang complèteront cette palette de signes révélateurs.

Le premier degré du récit est fait d'images naturalistes d'une grande précision photographique. Au ralenti et dans une sorte de halo créé par la surexposition apparaît l'imaginaire des personnages ou celui du cinéaste. Cette déréalisation hypothétique permet peut-être d'accepter les plus insupportables événements. Mais le plus dur pourrait bien être la mort accidentelle d'un enfant qui fait sombrer sa mère dans le désespoir, racontée par un poète hanté par la provocation qui cherche son accomplissement dans l'excès surréaliste!

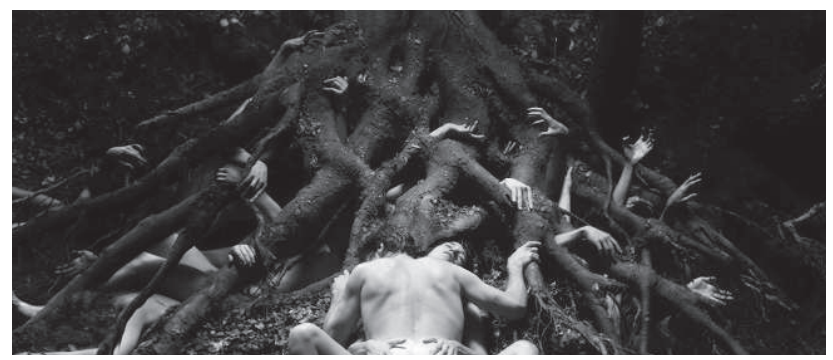

Ce choix de quatre films n'a bien sûr rien d'un échantillon scientifique. Il tient en partie du hasard. Il ne permet même pas de se demander si le Danemark est plus prolifique que la Suède, la Norvège ou la Finlande. Mais il correspond à des choix personnels.

Ces sueurs froides scandinaves tiennent plus de l'angoisse, qui trouble, étonne, dérange, que de la peur, qui fait sursauter. Pour faire peur, on utilise la violence, le sang qui gicle, la mort que l'on inflige sans raison à d'autres. Pour susciter l'angoisse, on suggère, on détourne ou on évite les grands gestes et les grands élans. Certains commencent avec la volonté d'intriguer et d'angoisser, pour virer de bord et faire peur gratuitement (Just Another Love Story) ou en tentant une justification par l'étrangeté (Sauna). Certes, Lars von Trier malmène quelque peu le spectateur dans les dernières minutes de son film: mais l'automutilation d'une femme amoureuse et culpabilisée par la mort de son fils, avec cette violence tournée contre elle-même, n'a pas le même poids que la cruauté pratiquée souvent gratuitement sur les autres. Et l'on en est ainsi revenu à l'adhésion au cinéma d'auteur après un détour par un genre. 


\section{Marchés scandinave et anglophone}

Certes, hier les films d'Ingmar Bergman étaient fort bien diffusés dans le monde entier. Il est probable qu'il en va de même, actuellement, de ceux de Lars von Trier. Mais pourquoi beaucoup d'autres n'atteignentils pas les marchés francophones ou les écrans ouverts de télévision? Une hypothèse que l'on entend parfois tiendrait à un manque de dynamisme des organisations scandinaves qui seraient surtout tournées vers une exploitation du marché intérieur élargi à l'Islande, la Finlande et au Danemark. Il ne reste alors pour le public francophone qui veut découvrir le cinéma scandinave, et pas seulement les productions de genre fantastique, les festivals et les petits circuits culturels qui ne sont plus très nombreux.

\section{Bergman, Lars von Trier et la télévision}

Un Bergman par exemple n'a jamais méprisé la télévision, lui qui était scénariste, réalisateur, producteur, écrivain et metteur en scène de théâtre. Car à l'origine, La Flûte enchantée (Suède, 1975), Après la répétition (Suède, 1984), En présence d'un clown (Suède, 1997), De la vie des marionnettes (Suède, 1980), Fanny et Alexandre (Suède, 1982) ou encore Sarabande (Suède, 2003) furent des téléfilms, parfois des débuts de séries ensuite retravaillées pour le grand écran. Il faut aussi rappeler que Lars von Trier fut assez bien accueilli sur les petits écrans avec sa série $L ' H \hat{o}^{-}$ pital et ses fantômes (Danemark, 1994-1997), déclinée en deux saisons et adaptée pour le marché américain par Stephen King en 2004 sous le titre de Kingdom Hospital (Etats-Unis, 2004). Des rapprochements furent parfois faits avec la série Urgences (ER, Michael Crichton, Etats-Unis, 1994-2009) tirant vers l'horreur, comparaison moins riche qu'avec Trin Peaks (Etats-Unis, 1990-1991) de David Lnych, une des révélations des années 1990. Un lien étroit unit les cinéastes scandinaves à leur marché télévisuel.

\section{Révolution dans l'audiovisuel: les séries "pointues"}

La notion d'auteur est problématisée par la pratique des séries télévisées, celui-ci étant en ce cas l'inventeur d'une idée destinée à être exploitée sur le long terme. Déclinée en de multiples épisodes par des équipes de scénaristes homogènes, interprétés par les mêmes acteurs d'un bout à l'autre, une série est mise en scène par des réalisateurs interchangeables qui occupent avant tout la fonction de techniciens. Néanmoins, les séries télévisées représentent indéniablement l'un des plus grands progrès accomplis par le langage audiovisuel qui se manifeste depuis le début de siècle à travers de nombreuses réussites américaines, produites 
par des chaînes à péage comme HBO. En 2006, le NIFFF a ouvert une riche fenêtre sur la série Masters of Horror (Mick Garris, Etats-Unis, 20052007), chaque épisode étant signé par un "maître» du genre (sur cette série, voir Laurent Guido, "NIFFF 2006. Les multiples visages de l'horreur contemporaine", Décadrages, n 8-9, automne 2006, pages 155-160). Tous formats confondus, le Homecoming (Etats-Unis, 2005) épisode réalisé par Joe Dante était l'un des meilleurs film d'"horreur» politique jamais réalisé, toutes origines confondues! Modestement, aux côtés de "Cinéma tout Ecran» à Genève, le NIFFF a salué l'apparition de ce nouvel élan créatif qui s'exprime par des séries "pointues" comme Les Soprano (David Chase, Etats-Unis, 1999-2007), Deadwood (David Milch, Etats-Unis 2004-2006), Six Feet Under (Alan Ball, Etats-Unis, 2001-2005) ou encore Roma (John Milius, William J. MacDonald, Bruno Heller, Etats-Unis/Grande-Bretagne/Italie, 2005-2007), qui n'ont pas encore été accueillies dans les festivals de cinéma, du moins à notre connaissance.

\section{Le NIFFF vu par...}

Dans une petite ville, alors que les cinéphiles «classiques» sont de moins en moins nombreux, on se connaît forcément, on se côtoie, sans nécessairement tout partager. L'auteur de ces lignes n'a aucune attirance particulière pour le fantastique s'il lorgne vers le gore pur, dur et gratuit, mais suffisamment de curiosité pour s'approcher des passions de proches. Il vaut alors la peine de découvrir le NIFFF à travers quatre d'entre ceux qui l'ont fait et le font vivre. Au départ, une idée d'entretiens qui partageraient la même structure, autour de cinq raisons de satisfaction et quelques regrets. L'alternance des questions/réponses a rapidement donné lieu à une conversation informelle, retranscrite librement ci-dessous sans recourir aux guillemets d'usage.

\section{1/ Olivier Müller, fondateur et ancien président}

L'attirance pour le cinéma de genre tient peut-être à un défi d'enfance quand, à douze ans et contre le gré parental, Olivier découvre, en cachette, Massacre à la tronçonneuse (Texas Chainsaw Massacre, Hooper, Etats-Unis, 1974)! Quinze ans plus tard, ses études achevées, Olivier Müller rêve de créer dans sa région natale, avec quelques amis, un festival où il aurait aimé se rendre en spectateur. Une association est fondée en 1998 ; la première mouture du NIFFF voit le jour en 2000.

Dans le sillage de Marco Müller qui a permis de découvrir à Locarno de nombreux aspects du cinéma asiatique, le NIFFF accorde une place de plus en plus importante à l'Asie sans omettre la dimension fantastique, ni s'en tenir uniquement au Japon. Le coréen Kim Ki Duk n'était 
pas le seul à réaliser de bons films. Mais il fallut apprendre à faire coexister harmonieusement la chèvre du genre avec le chou de la politique des auteurs, un exercice que le NIFFF pratique naturellement et avec bonheur.

Le cinéma scandinave fantastique a une réputation dans l'histoire à travers de grands auteurs. Un Lars von Trier est à présent mondialement connu. Derrière lui, le foisonnement est intense, mais avec des films qui souvent restent confinés sur les marchés intérieurs. Le NIFFF se devait de contribuer à les faire mieux connaître.

En 2007, Olivier Müller entre à la Section du cinéma de l'OFC. Il prend naturellement ses distances avec le NIFFF, satisfait de constater que le succès se confirme, que le fantastique est toujours décliné sous toutes ses formes, que l'Open Air est enfin mis en place, que la gestion reste rigoureuse et que le festival mieux reconnu est donc d'avantage soutenu. Il peut alors se souvenir des rencontres prolongées hors projections avec un Ray Harryhausen, un Brian Aldiss qui expliquait à Kubrick qu'il était incapable d'adapter son A.I. et tant d'autres, tout surpris d'être à l'aise avec des personnalités du troisième âge qui ont marqué l'Histoire du cinéma tout court. Et puis, en 2009, pour la première fois depuis que le festival existe, il s'est offert un luxe : voir un film sur l'écran, dans une salle, avec le public et dans sa totalité.

\section{2/ Anaïs Emery, cofondatrice et directrice artistique}

Anaïs Emery, directrice artistique, est aussi de la première heure du NIFFF, dès la fondation de l'Association. Bien sûr, elle a commencé par un bénévolat total. Elle est maintenant salariée par le NIFFF avec un contrat annuel à quatre-vingt pourcents.

Anaïs Emery relève cinq points forts.

1/ Avoir osé choisir une ville d'un peu plus de 30000 habitants pour y installer une manifestation culturelle "pointue», attirant aussi beaucoup de jeunes suisses alémaniques, Neuchâtel n’étant pas très éloigné de la frontière linguistique. Y aurait-t-il suffisamment de curieux pour accepter de voir toutes sortes de films dans une manifestation consacrée à un genre précis? D’emblée, une partie des «fans» qui ne s'inscrivent pas parmi les intellectuels adeptes de la politique des auteurs répondit présent!

2/ Produire chaque année une rétrospective par les seuls moyens du bord demande effort et patience pour trouver les copies, les réunir et les présenter, parfois les sous-titrer. Ainsi fut fait!

3/ Pouvoir se féliciter, à titre personnel et pour les participants, de rencontres faites avec des invités ou membres du jury, par exemple le 
japonais Shynia Tsukamato, qui a occupé un peu tous les postes disponibles dans le cinéma, ou Tob Hopper, auteur d'un film culte, Massacre à la tronçonneuse, qui oblige à se souvenir que le son est aussi un moyen efficace à mettre au service du fantastique.

4/ La possibilité d'être parvenus, au fur et à mesure, à concrétiser une bonne partie de leurs désirs, tels que l'Open Air, bien en place en 2009, qui attire le public local.

5/ Savoir enfin que la légitimité du festival est reconnue par la Section du cinéma de l'OFC, reconnaissance amorcée dès 2005 sous l'ère Wehrlin, confirmée ensuite par Nicolas Bideau avec un contrat de prestations.

Restent qu'à ces raisons de satisfaction s'ajoutent tout de même des soucis concernant la relève des équipes de la première décennie, des incertitudes sur le financement, la timidité des distributeurs qui n'achètent pas (pas encore ?) des films montrés par le NIFFF - même s'ils en profitent pour montrer certains films de leurs catalogues en avant-première - et un serpent de mer, celui du choix des dates de la manifestation.

\section{3/ Pierre-Yves Jeanneret, cofondateur et actuel président}

En 1998, assistant à l'Université de Neuchâtel, Pierre-Yves Jeanneret fait partie de l'association qui rêve de créer le NIFFF et dont la curiosité du genre a été nourrie par la revue Mad Movies. En cinq dates ou périodes, il survole le festival.

- 2000 : le rêve prend forme, dans les trois salles de l'Apollo. Un public existe, prêt à partager des découvertes, déjà orientées vers l'Asie. Il faut tout apprendre, dans un climat de bénévolat généralisé.

- 2002: 2001 a permis d'effacer les dettes de la première édition. Les "fans» sont présents, mais ce ne sont pas des enfants de 1968. Le film culte Massacre à la tronçonneuse peut aussi être considéré, avec du recul, comme une protestation contre la guerre du Vietnam.

- 2003: une immense personnalité impose sa présence, l'inventeur de formes cinématographiques originales, qui finit par faire oublier le cinéaste qui signe les films auxquels il participe. Ray Harryhausen est parfois le véritable "auteur» de films auxquels il apporte sa collaboration.

Le succès se confirme. Que la moitié des spectateurs qui assistent à certaines projections prenne la peine de répondre en vue du prix du public est un signe très positif. Le travail effectué par une étudiante dans le cadre de sa formation, qui cherche à comprendre de qui se compose ce public, mérite grande attention pour mieux cerner les espoirs des participants. 
- 2004/2005 : l'assistant universitaire entre au service de la statistique de l'Etat: le temps de bénévolat se réduit. Il faudra traiter au moins le dossier des Méliès. L'arrivée de Michel Vust comme codirecteur renforce l'organisation d'une manière professionnelle. Le budget de CHF 750 000.- reste couvert pour un tiers par le bénévolat.

- 2006/2009: le NIFFF continue sa progression. L'offre annuelle s'approche de la centaine de séances en quatre puis cinq jours. Le Théâtre du Passage devient le centre névralgique du festival. Il faut un successeur à Olivier Müller parti pour Berne. Pierre-Yves Jeanneret se charge de cette fonction qui est tout sauf seulement honorifique.

Aux éléments positifs qui précèdent s'ajoutent des regrets qui sont autant d'espoirs pour mettre en place d'indispensables améliorations. Une année sur deux, le NIFFF connaît la concurrence du football, européen ou mondial. Pour éviter cette "concurrence», la retransmission d'une finale sur grand écran inscrite au programme touche au fantastique! Des moyens sont mis en œuvre pour mieux connaitre la composition d'un public aux aspirations variées.

Une pointe d'angoisse: le fantastique semble de plus en plus se laisser gagner par la surcharge, occultant sur le fond.

\section{4/ Mathieu Béguelin, présentateur}

Dans une manifestation aux sources multiples, entre autres asiatiques, les versions originales sont de rigueur, si possible sous-titrées. Mais elles le sont, souvent, en anglais. Le festival organise des sous-titrages par projection sur un écran adjacent. "Passeur» entre l'œuvre ou l'invité venu de loin et le spectateur, Mathieu Béguelin situe les films sans prononcer de jugement de valeur avant leur projection ou pour faciliter la compréhension de la discussion avec les invités qui suit.

Le présentateur sent bien qu'il n'y a pas un mais des publics. Celui qui veut savoir comment fonctionne la censure à Hong-Kong n'attend pas la même chose que les adeptes des courts métrages suisses. Une particularité du NIFFF: l'insatisfaction ne vide pas les salles! Le public y est même parfois d'une infinie patience devant certains incidents.

Lors des débats, il ne s'agit par seulement de traduire, mais aussi d'interpréter les réponses, comme cela s'est par exemple produit avec Terry Gilliam. Il faut parfois contribuer à mettre à l'aise une invitée d'une incroyable timidité, telle Lucile Hadzihalilovic l'année où elle obtint une grand prix. Le présentateur se souvient de la gentillesse d'un George A. Romero, la passion d'un John Landis, la complicité entre le passeur et l'invité se prolongeant hors de la salle de projection. 


\section{Conclusion}

Il eut été possible de traiter d'autres aspects du NIFFF 2009, par exemple le rôle joué par la section "Open air» ou les informations apportées sur la «censure» à Hong-Kong à travers la "Catégorie III : Transgression". Un examen attentif de la manière dont le NIFFF s'est ouvert dès sa création au court-métrage fantastique dans le sens le plus large, tant suisse qu'européen, eut permis de prendre acte des promesses tenues par le format court qui n'est pas toujours mis en valeur.

Enfin, nous aurions pu justifier plus précisément les raisons de notre adhésion aux séries télévisées contemporaines, emmenées par les EtatsUnis et les besoins des chaînes à péage, que nous considérons comme véritablement révolutionnaires.

On retrouvera dans les mois qui viennent certains de ces développements sur le site www. decadrages.ch. 